\title{
An Infrared Spectroscopy Study of the Conformational Evolution of the Bis(trifluoromethanesulfonyl)imide Ion in the Liquid and in the Glass State
}

\author{
Oriele Palumbo, ${ }^{1}$ Francesco Trequattrini, ${ }^{2}$ Francesco Maria Vitucci, ${ }^{1}$ \\ Maria Assunta Navarra, ${ }^{3}$ Stefania Panero, ${ }^{3}$ and Annalisa Paolone ${ }^{1}$ \\ ${ }^{1}$ CNR-ISC, UOS La Sapienza, Piazzale Aldo Moro 5, 00185 Roma, Italy \\ ${ }^{2}$ Dipartimento di Fisica, Sapienza Università di Roma, Piazzale Aldo Moro 5, 00185 Roma, Italy \\ ${ }^{3}$ Dipartimento di Chimica, Sapienza Università di Roma, Piazzale Aldo Moro 5, 00185 Roma, Italy \\ Correspondence should be addressed to Annalisa Paolone; annalisa.paolone@romal.infn.it
}

Received 9 April 2015; Revised 16 July 2015; Accepted 26 July 2015

Academic Editor: Fajun Zhang

Copyright (c) 2015 Oriele Palumbo et al. This is an open access article distributed under the Creative Commons Attribution License, which permits unrestricted use, distribution, and reproduction in any medium, provided the original work is properly cited.

\begin{abstract}
We measure the far-infrared spectrum of N,N-Dimethyl-N-ethyl-N-benzylammonium (DEBA) bis(trifluoromethanesulfonyl)imide (TFSI) ionic liquid (IL) in the temperature range between 160 and $307 \mathrm{~K}$. Differential scanning calorimetry measurements indicate that such IL undergoes a glass transition around $210 \mathrm{~K}$. DFT calculations allow us to assign all the experimental absorptions to specific vibrations of the DEBA cation or of the two conformers of the TFSI anion. We find that the vibration frequencies calculated by means of the PBE0 functional are in better agreement with the experimental ones than those calculated at the B3LYP level, largely used for the attribution of vibration lines of ionic liquids. Experimentally we show that, in the liquid state, the relative concentrations of the two conformers of TFSI depend on temperature through the Boltzmann factor and the energy separation, $\Delta H$, is found to be $\approx 2 \mathrm{~kJ} / \mathrm{mol}$, in agreement with previous calculations and literature. However, in the glassy state, the concentrations of the cis-TFSI and trans-TFSI remain fixed, witnessing the frozen state of this phase.
\end{abstract}

\section{Introduction}

Ionic liquids (ILs) are molten salts with melting temperatures lower than $373 \mathrm{~K}$ and are typically composed of organic cations, like imidazolium, pyrrolidinium, ammonium, or alkyl phosphonium, and organic/inorganic anions, like hexafluorophosphate, tetrafluoroborate, triflate, dicyanamide, tetracyanamethanide, or bis(trifluoromethanesulfonyl)imide (TFSI). They have large applicative potentialities in the fields of catalysis and green chemistry and as solvents and electrolytes lubricants and ingredients for pharmaceuticals and heat exchangers [1-5].

From a fundamental point of view, ionic liquids are quite intriguing, as they present extremely rich phase diagrams as a function of temperature and pressure. For example, on cooling, N-butyl-N-methyl-pyrrolidinium bis(trifluoromethanesulfonyl)imide ( $\mathrm{PYR}_{14}$ TFSI) displays a large undercooled liquid region and transforms into a glass around
$190 \mathrm{~K}$. On heating, the glass transforms into a supercooled liquid and around $217 \mathrm{~K}$ it crystallizes. On further heating a solid-solid phase transition is revealed around $241 \mathrm{~K}$ and finally the sample melts at $\sim 268 \mathrm{~K}$ $[6,7]$. In PYR $_{14}$-per(fluoroalkylsulfonyl)imide ionic liquids one can observe a sharp solid-liquid transition in case of bis(trifluoromethanesulfonyl)imide and (pentafluoroethanesulfonyl)(nonafluorobutanesulfonyl)imide, but only a glass transition is present in the low temperature DSC traces of (nonafluorobutanesulfonyl)(toluenesulfonyl)imide [8]. Also the behavior of ILs as a function of pressure is extremely dependent on the specific liquid. For instance, in analogy with cold crystallization usually observed when supercooled ionic liquids are reheated, high pressure Raman experiments showed that two different ionic liquids based on the $\left[\mathrm{BF}_{4}\right]^{-}$anion can be pressurized above $5.0 \mathrm{GPa}$ at room temperature, undergoing crystallization after releasing the 
pressure below $\sim 2.0 \mathrm{GPa}$ [9-13]. Moreover, N,N-diethyl-Nmethyl-N-(2-methoxyethyl)ammonium bis(trifluoromethylsulfonyl)imide does not crystallize up to $5.0 \mathrm{GPa}$ at room temperature, but a pressure-induced change in the structural organization might occur around this pressure range [9-12]. Other studies detected below 1.0 GPa the presence of liquidcrystal and crystal phase transitions of imidazolium based ILs, having different anion, that is, $\mathrm{Cl}^{-}, \mathrm{Br}^{-}$, and $\mathrm{PF}_{6}{ }^{-}[14,15]$.

Moreover, many of the ions composing the ILs have geometric isomers, differing in the orientation of chemical bonds. Usually, conformers coexist in the liquid state according to the Boltzmann distribution, such as in the case of $\mathrm{PYR}_{14}$ TFSI [16]. However, in the solid state, in many cases only the lowest energy conformer survives, but more complex situations have been reported. Indeed, in N-trimethyl-N-propylammonium bis(trifluoromethanesulfonyl)imide (TMPATFSI) both conformers of TFSI are present in solid state, while, by changing the length of the alkyl chain, only the higher energy conformer of this ion (cis-TFSI) is observed in the solid state of $\mathrm{N}$-trimethyl-N-hexylammonium bis(trifluoromethanesulfonyl)imide (TMHA-TFSI) [17]. To the best of our knowledge, no detailed investigation of the glass state as a function of temperature exists.

For some ions, conformers have been identified by means of spectroscopic techniques, such as Raman and infrared spectroscopy, combined with ab initio or DFT calculations of the vibration frequencies. A large effort has been devoted to the spectroscopic investigation of the conformers of TFSI by means of Raman and infrared spectroscopy [16-21]. It possesses two conformers differing in energy by only $2.2 \mathrm{KJ} / \mathrm{mol}$ [16-21]. The Raman spectrum displays easily detectable lines centred around $629 \mathrm{~cm}^{-1}$ for the transoid form and at $\sim 330$ and $653 \mathrm{~cm}^{-1}$ for the cisoid conformer $[16,18-20]$. Furthermore, trans-TFSI displays a strong infrared absorption around $628 \mathrm{~cm}^{-1}$, while cis-TFSI shows two lines centered at $\sim 602$ and $650 \mathrm{~cm}^{-1}[17,21]$. Another example of synergy between spectroscopic and computational techniques is that of bis(fluorosulfonyl) imide [22]. For this ion two conformers with cis- and trans- structure were identified.

It is worth noting that most DFT calculations performed on ionic liquids are usually performed by the B3LYP functional and the $6-31 \mathrm{G}^{* *}$ basis set. Indeed, a quite good agreement between the calculated and experimental vibration lines of ionic liquids is obtained. To the best of our knowledge, only in one extreme case was a different choice of the basis set used: indeed, for the bis(fluorosulfonyl) imide the 6-311+G (3df) basis set was used, maintaining, however, the B3LYP functional [22]. It must be noted that calculations with such a big basis set are extremely time consuming and cannot be routinely performed. In the case of the FSI ion, the shift of experimental vibration bands and the lines calculated at the B3LYP/6-31G** is about $10 \%$ and therefore a better model had to be found.

Spectroscopic investigation can provide information about the relative concentrations of conformers. Indeed, the ratio of the intensities, $r$, of the lines attributable to the different rotamers is proportional to their relative concentration. From the changes of $r$ as a function of temperature, one can verify the Boltzmann distributions of the concentrations of conformers and calculate the energy difference between the rotamers $[16,23]$.

In the present paper we investigate the infrared absorption spectrum of the N,N-Dimethyl-N-ethyl-N-benzylammonium bis(trifluoromethanesulfonyl)imide (DEBA-TFSI) ionic liquid. For comparison, we consider previous results obtained on N-trimethyl-N-propylammonium bis(trifluoromethanesulfonyl)imide (TMPA-TFSI) and N-trimethyl-N-hexylammonium bis(trifluoromethanesulfonyl)imide (TMHA-TFSI), which share the same anion (TFSI) and have an ammonium based cation, too. Indeed TMPA and TMHA differ in the length of the alkyl chain, whereas in DEBA a close benzyl ring is present. While TMPA-TFSI and TMHA-TFSI crystallize at low temperature, we show that DEBA-TFSI undergoes a glass transition around $210 \mathrm{~K}$ when cooled with a temperature rate of $5 \mathrm{~K} / \mathrm{min}$.

DFT calculations allow the attribution of the measured infrared lines to the vibrations of the ions. In the present paper we explored the potentialities of two other functionals (B97-D and PBE0) to reproduce the experimental vibration bands. We show that PBE0 is able to reproduce experimental frequencies even better than B3LYP, without increments in the computation time.

Once the attribution of vibration lines is settled by the comparison of calculated and experimental bands, we show that in the liquid state the relative concentration of the two conformers of TFSI follows the Boltzmann law, but, on entering the glass state, the distribution of the conformer population is completely frozen.

\section{Materials and Methods}

2.1. Experimental. The presently measured ionic liquids N,N-Dimethyl-N-ethyl-N-benzylammonium bis(trifluoromethanesulfonyl)imide (DEBA-TFSI) (purity > 99.5\%), $\mathrm{N}$-trimethyl-N-propylammonium bis(trifluoromethanesulfonyl)imide (TMPA-TFSI) (purity > 99.9\%), and N-trimethyl-N-hexylammonium bis(trifluoromethanesulfonyl)imide (TMHA-TFSI) (purity > 99.9\%) were purchased from Solvionic and used as received.

Differential scanning calorimetry (DSC) measurements were performed by means of a Mettler-Toledo DSC 821, under an inert nitrogen flux, cooling from $315 \mathrm{~K}$ down to $160 \mathrm{~K}$ and then heating back to $315 \mathrm{~K}$ with a temperature rate of $5 \mathrm{~K} / \mathrm{min}$.

Infrared spectroscopy measurements were performed by means of a Bruker Vertex 125 HR spectrometer at the AILES beamline of Soleil Synchrotron [24, 25]. For measurements in the region below $670 \mathrm{~cm}^{-1}$ the interferometer was equipped with a $6 \mu$ Mylar beamsplitter and a bolometer detector. The resolution of spectra was $0.5 \mathrm{~cm}^{-1}$. Thin layers of ionic liquids were placed between two polyethylene windows for measurements in the far-infrared region. The transmission was recorded, using as a reference the spectrum of the optical windows. Transmission measurements were converted to absorbance data, as usual. The samples were cooled down to $160 \mathrm{~K}$ by means of a Cryomac cryopump with a temperature rate of $\sim 5 \mathrm{~K} / \mathrm{min}$ and data were collected on heating between this minimum temperature and $307 \mathrm{~K}$. 


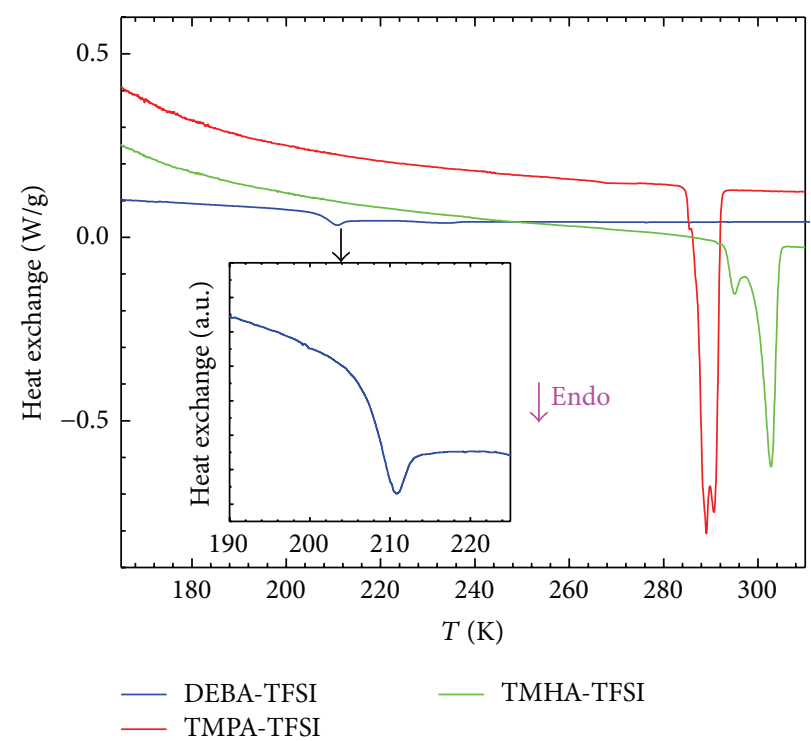

FIGURE 1: DSC curves of DEBA-TFSI measured at $5 \mathrm{~K} / \mathrm{min}$ on heating. For comparison the curves of TMPA-TFSI and TMHA-TFSI measured in the same conditions are reported.

2.2. Computational. Preliminary DFT calculations were performed using the Spartan software $[26,27]$ to find the geometry of N,N-Dimethyl-N-ethyl-N-benzylammonium (DEBA) and bis(trifluoromethanesulfonyl)imide (TFSI) ions. For DEBA we considered one geometry only, while for the TFSI ion we investigated both the cisoid and transoid conformers. We optimized the structure of each ion by means of the Firefly package $[28,29]$ and then we calculate the hessian matrix, the vibration frequencies, and the IR intensities. Various combinations of theory and basis sets were used. We started from the B3LYP hybrid density functional theory methods, adopting the $6-31 \mathrm{G}^{* *}$ basis set, as this particular choice of basis set and theory was extensively used in previous literature in order to calculate the infrared and Raman spectra of ions composing ILs [16-21]. A previous investigation of the TMPA and TMHA cations performed at the B3LYP/6-31G ${ }^{* *}$ level already attributed the infrared lines of these ions $[17,21]$ and therefore we did not repeat those calculations. Moreover, we extended our study of the ionic vibrations of DEBA, cisTFSI, and trans-TFSI to the following combinations of theory and basis sets in order to check a proper attribution of the experimental lines: B3LYP/6-311G ${ }^{* *}, \mathrm{~B} 97-\mathrm{D} / 6-31 \mathrm{G}^{* *}$, and PBE0/6-31G ${ }^{* *}$. For each ion, the IR spectrum was simulated by summing Lorentzian curves centered at each calculated IR vibration frequency with a fixed $10 \mathrm{~cm}^{-1}$ peak width.

\section{Results and Discussion}

3.1. DSC Measurements: Identification of the Glass Transition. The DSC curve of DEBA-TFSI, measured on heating at $5 \mathrm{~K} / \mathrm{min}$, is reported in Figure 1 . For comparison we report also the DSC curves of TMPA-TFSI and TMHATFSI, acquired in the same conditions. The latter two ionic liquids are known to undergo solid crystalline to liquid transition around 295 and $300 \mathrm{~K}$ [17], respectively. Indeed, DSC measurements of these compounds display intense endothermic peaks between 282 and $293 \mathrm{~K}$ in TMPA-TFSI and between 291 and $306 \mathrm{~K}$ in TMHA-TFSI (see Figure 1). These peaks are structured and large [17]. The integral of the peaks gives the latent heat of liquefaction, which is found to be $57.3 \mathrm{~J} / \mathrm{g}$ for TMPA-TFSI and $39.0 \mathrm{~J} / \mathrm{g}$ for TMHA-TFSI. On the contrary, DEBA-TFSI shows a much less intense thermal transition between 206 and $214 \mathrm{~K}$, whose integral is as low as $1.0 \mathrm{~J} / \mathrm{g}$. Moreover, the latter transition has a different shape from that due to liquefaction from a crystalline state and resembles the shape of a glass transition. It must be noted that the value of the latent heat of TMPA-TFSI and TMHA-TFSI is comparable to that corresponding to the melting of the crystalline state of 1-butyl-1-methylpyrrolidinium bis(trifluoromethanesulfonyl)imide (PYR $\left.{ }_{14} \mathrm{TFSI}\right)(55.8 \mathrm{~J} / \mathrm{g})$ [7], which is considered an archetypal ionic liquid. Conversely, the integral of the endothermic peak of DEBA-TFSI is comparable to the value measured in $\mathrm{PYR}_{14}$ TFSI, when passing from the glass to the liquid state $(0.48 \mathrm{~J} / \mathrm{g})$ [7]. Therefore, the present measures prove that, on cooling at $5 \mathrm{~K} / \mathrm{min}$ DEBA-TFSI, one observes a glassy state at low temperatures (below $\sim 210 \mathrm{~K}$ ) and, remarkably, no other thermal features, neither melting nor crystallization, appear above $210 \mathrm{~K}$. This is a very favorable property for an ionic liquid compound, being liquid in an extended temperature region, at subzero conditions.

3.2. DFT Calculations of the Vibration Frequencies. The optimized geometries of trans-TFSI, cis-TFSI, TMPA, TMHA, and DEBA at the B3LYP/6-31G ${ }^{* *}$ level are shown in Figure 2. The unscaled calculated frequency and intensity of the vibrations of the single ions trans-TFSI, cis-TFSI, and DEBA in the spectral range between 150 and $665 \mathrm{~cm}^{-1}$ are reported 
TABLE 1: Unscaled calculated frequency and intensity of the vibrations of trans-TFSI in the frequency range between 150 and $670 \mathrm{~cm}^{-1}$, with various levels of theory and basis sets.

\begin{tabular}{|c|c|c|c|c|c|c|c|}
\hline \multicolumn{2}{|c|}{ B3LYP/6-31G ${ }^{* *}$} & \multicolumn{2}{|c|}{ B3LYP/6-311G ${ }^{* *}$} & \multicolumn{2}{|c|}{ B97-D/6-31G ${ }^{* *}$} & \multicolumn{2}{|c|}{ PBE0/6-31G ${ }^{* *}$} \\
\hline $\begin{array}{l}\text { Frequency } \\
\left(\mathrm{cm}^{-1}\right)\end{array}$ & $\begin{array}{l}\text { Intensity } \\
(\mathrm{km} / \mathrm{mol})\end{array}$ & $\begin{array}{c}\text { Frequency } \\
\left(\mathrm{cm}^{-1}\right)\end{array}$ & $\begin{array}{l}\text { Intensity } \\
(\mathrm{km} / \mathrm{mol})\end{array}$ & $\begin{array}{l}\text { Frequency } \\
\left(\mathrm{cm}^{-1}\right)\end{array}$ & $\begin{array}{l}\text { Intensity } \\
(\mathrm{km} / \mathrm{mol})\end{array}$ & $\begin{array}{l}\text { Frequency } \\
\left(\mathrm{cm}^{-1}\right)\end{array}$ & $\begin{array}{l}\text { Intensity } \\
(\mathrm{km} / \mathrm{mol})\end{array}$ \\
\hline 155 & 0.2 & 154 & 0.2 & 153 & 0.2 & 157 & 0.2 \\
\hline 189 & 2.4 & 189 & 2.5 & 182 & 1.7 & 191 & 2.5 \\
\hline 197 & 0.5 & 196 & 0.6 & 189 & 0.4 & 198 & 0.5 \\
\hline 217 & 10.2 & 215 & 9.7 & 207 & 7.4 & 220 & 10.4 \\
\hline 262 & 0.1 & 257 & 0.1 & 241 & 0.1 & 270 & 0.1 \\
\hline 275 & 0.4 & 276 & 0.4 & 260 & 0.3 & 281 & 0.5 \\
\hline 295 & 0.2 & 293 & 0.1 & 274 & 0.1 & 304 & 0.3 \\
\hline 304 & 0.4 & 296 & 0.7 & 277 & 1.0 & 316 & 0.3 \\
\hline 325 & 0.2 & 323 & 0.2 & 308 & 0.1 & 332 & 0.2 \\
\hline 342 & 3.3 & 340 & 3.7 & 326 & 4.8 & 351 & 3.4 \\
\hline 376 & 4.9 & 374 & 5.1 & 356 & 3.6 & 387 & 5.6 \\
\hline 391 & 5.0 & 390 & 5.9 & 373 & 2.7 & 400 & 5.7 \\
\hline 497 & 73.8 & 497 & 79.9 & 477 & 65.8 & 506 & 75.9 \\
\hline 519 & 0.0 & 519 & 0.0 & 497 & 0.1 & 529 & 0.0 \\
\hline 536 & 1.4 & 536 & 1.3 & 514 & 0.5 & 548 & 1.5 \\
\hline 544 & 0.3 & 544 & 0.4 & 521 & 0.3 & 556 & 0.2 \\
\hline 557 & 51.2 & 555 & 52.1 & 533 & 49.2 & 569 & 51.6 \\
\hline 570 & 0.0 & 569 & 0.0 & 542 & 0.0 & 585 & 0.0 \\
\hline 602 & 343.2 & 601 & 361.5 & 569 & 302.5 & 619 & 345.2 \\
\hline 608 & 3.1 & 604 & 5.0 & 582 & 1.0 & 622 & 4.5 \\
\hline
\end{tabular}

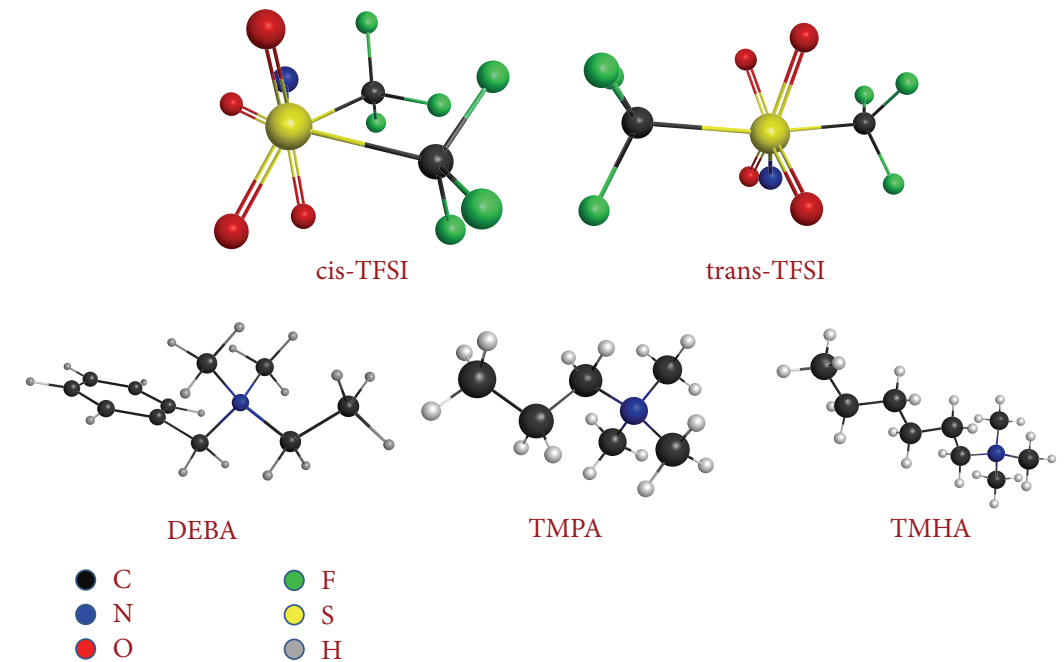

FIgURE 2: Geometry of cis-TFSI, trans-TFSI, TMPA, TMHA, and DEBA.

in Tables 1-3, respectively, for the various theory/basis set combinations. Panels (a)-(d) of Figures 3 and 4 display the infrared spectrum of the single ions (trans-TFSI, cisTFSI, and DEBA), calculated by summing $10 \mathrm{~cm}^{-1}$ width Lorentzian curves centered at each computed IR vibration frequency. At the B3LYP/6-31G ${ }^{* *}$ level it is known that, for the two TFSI conformers, a scaling factor of 1.040 gives a good coincidence of the calculated vibration frequencies with the experimental lines, as already reported in [1621]. For the other combinations of theories and basis set information is not available. For the DEBA ion, no other comparison between the calculated and experimental data has been reported so far. In the following, we compare the most intense absorption lines obtained from experiments with those obtained by the DFT calculations. We will discuss the results obtained by means of the various theories and 
TABLE 2: Unscaled calculated frequency and intensity of the vibrations of cis-TFSI in the frequency range between 150 and $670 \mathrm{~cm}^{-1}$, with various levels of theory and basis sets.

\begin{tabular}{|c|c|c|c|c|c|c|c|}
\hline \multicolumn{2}{|c|}{ B3LYP/6-31G ${ }^{* *}$} & \multicolumn{2}{|c|}{ B3LYP/6-311G ${ }^{* *}$} & \multicolumn{2}{|c|}{ B97-D/6-31G ${ }^{* *}$} & \multicolumn{2}{|c|}{$\mathrm{PBE} 0 / 6-31 \mathrm{G}^{* *}$} \\
\hline $\begin{array}{l}\text { Frequency } \\
\left(\mathrm{cm}^{-1}\right)\end{array}$ & $\begin{array}{c}\text { Intensity } \\
(\mathrm{km} / \mathrm{mol})\end{array}$ & $\begin{array}{c}\text { Frequency } \\
\left(\mathrm{cm}^{-1}\right)\end{array}$ & $\begin{array}{c}\text { Intensity } \\
(\mathrm{km} / \mathrm{mol})\end{array}$ & $\begin{array}{c}\text { Frequency } \\
\left(\mathrm{cm}^{-1}\right)\end{array}$ & $\begin{array}{c}\text { Intensity } \\
(\mathrm{km} / \mathrm{mol})\end{array}$ & $\begin{array}{c}\text { Frequency } \\
\left(\mathrm{cm}^{-1}\right)\end{array}$ & $\begin{array}{c}\text { Intensity } \\
(\mathrm{km} / \mathrm{mol})\end{array}$ \\
\hline 154 & 1.5 & 160 & 1.6 & 152 & 1.3 & 155 & 1.5 \\
\hline 193 & 1.1 & 196 & 0.8 & 185 & 0.8 & 193 & 0.9 \\
\hline 193 & 0.1 & 199 & 0.7 & 185 & 0.0 & 194 & 0.3 \\
\hline 197 & 1.5 & 202 & 1.5 & 189 & 1.0 & 197 & 1.8 \\
\hline 265 & 0.3 & 265 & 0.3 & 245 & 0.3 & 273 & 0.5 \\
\hline 269 & 2.2 & 269 & 1.7 & 246 & 0.8 & 278 & 2.6 \\
\hline 292 & 0.5 & 295 & 0.4 & 276 & 0.2 & 299 & 0.5 \\
\hline 310 & 0.2 & 310 & 0.1 & 288 & 0.2 & 320 & 0.2 \\
\hline 316 & 0.7 & 315 & 0.7 & 296 & 0.7 & 325 & 0.5 \\
\hline 336 & 0.1 & 338 & 0.1 & 318 & 0.1 & 343 & 0.1 \\
\hline 383 & 4.9 & 384 & 5.0 & 364 & 3.6 & 393 & 5.9 \\
\hline 409 & 7.7 & 409 & 8.0 & 388 & 5.1 & 420 & 8.4 \\
\hline 496 & 86.2 & 496 & 88.8 & 476 & 78.3 & 505 & 89.3 \\
\hline 516 & 6.1 & 517 & 6.2 & 495 & 5.7 & 526 & 6.1 \\
\hline 535 & 1.1 & 535 & 0.9 & 514 & 0.7 & 547 & 1.2 \\
\hline 546 & 1.9 & 546 & 1.3 & 523 & 1.3 & 556 & 1.9 \\
\hline 557 & 40.1 & 556 & 38.8 & 533 & 30.3 & 569 & 43.3 \\
\hline 565 & 60.7 & 565 & 60.3 & 539 & 69.6 & 577 & 55.2 \\
\hline 582 & 128.9 & 583 & 130.0 & 551 & 117.6 & 597 & 127.4 \\
\hline 630 & 90.8 & 629 & 91.1 & 601 & 67.6 & 647 & 107.8 \\
\hline
\end{tabular}

TABLE 3: Unscaled calculated frequency and intensity of the vibrations of DEBA in the frequency range between 150 and $670 \mathrm{~cm}^{-1}$, with various levels of theory and basis sets.

\begin{tabular}{|c|c|c|c|c|c|c|c|}
\hline \multicolumn{2}{|c|}{ B3LYP/6-31G ${ }^{* *}$} & \multicolumn{2}{|c|}{ B3LYP/6-311G ${ }^{* *}$} & \multicolumn{2}{|c|}{ B97-D/6-31G ${ }^{* *}$} & \multicolumn{2}{|c|}{$\mathrm{PBE} 0 / 6-31 \mathrm{G}^{* *}$} \\
\hline $\begin{array}{l}\text { Frequency } \\
\left(\mathrm{cm}^{-1}\right)\end{array}$ & $\begin{array}{c}\text { Intensity } \\
(\mathrm{km} / \mathrm{mol})\end{array}$ & $\begin{array}{c}\text { Frequency } \\
\left(\mathrm{cm}^{-1}\right)\end{array}$ & $\begin{array}{c}\text { Intensity } \\
(\mathrm{km} / \mathrm{mol})\end{array}$ & $\begin{array}{c}\text { Frequency } \\
\left(\mathrm{cm}^{-1}\right)\end{array}$ & $\begin{array}{c}\text { Intensity } \\
(\mathrm{km} / \mathrm{mol})\end{array}$ & $\begin{array}{c}\text { Frequency } \\
\left(\mathrm{cm}^{-1}\right)\end{array}$ & $\begin{array}{c}\text { Intensity } \\
(\mathrm{km} / \mathrm{mol})\end{array}$ \\
\hline 223 & 2.7 & 222 & 2.7 & 234 & 2.4 & 227 & 2.8 \\
\hline 231 & 0.0 & 229 & 0.0 & 239 & 0.0 & 232 & 0.0 \\
\hline 268 & 1.6 & 267 & 1.7 & 269 & 1.1 & 273 & 2.2 \\
\hline 272 & 0.7 & 271 & 0.6 & 278 & 0.6 & 275 & 0.7 \\
\hline 305 & 3.3 & 305 & 2.2 & 317 & 2.5 & 313 & 3.3 \\
\hline 327 & 0.4 & 325 & 0.4 & 365 & 0.4 & 333 & 0.4 \\
\hline 358 & 1.4 & 357 & 0.9 & 389 & 0.0 & 364 & 1.6 \\
\hline 378 & 0.1 & 378 & 0.2 & 393 & 1.8 & 380 & 0.1 \\
\hline 415 & 0.0 & 413 & 0.0 & 411 & 0.1 & 419 & 0.1 \\
\hline 419 & 0.1 & 419 & 0.1 & 415 & 0.2 & 419 & 0.1 \\
\hline 443 & 0.4 & 440 & 0.3 & 442 & 0.5 & 447 & 0.4 \\
\hline 470 & 2.0 & 470 & 2.9 & 467 & 2.3 & 473 & 3.4 \\
\hline 550 & 5.5 & 552 & 6.9 & 540 & 6.6 & 554 & 4.5 \\
\hline 613 & 3.0 & 615 & 3.2 & 597 & 3.6 & 617 & 3.8 \\
\hline 633 & 0.1 & 638 & 0.1 & 618 & 0.1 & 631 & 0.1 \\
\hline
\end{tabular}




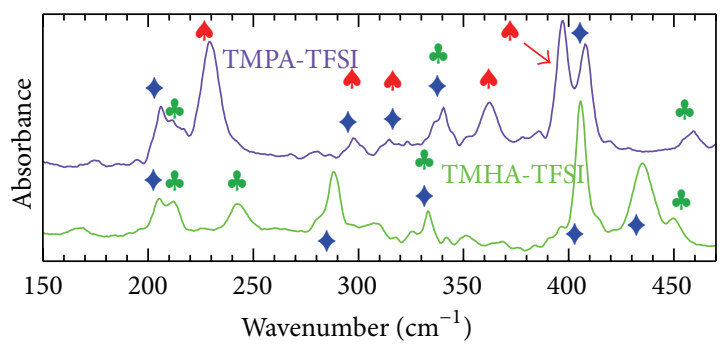

(f)

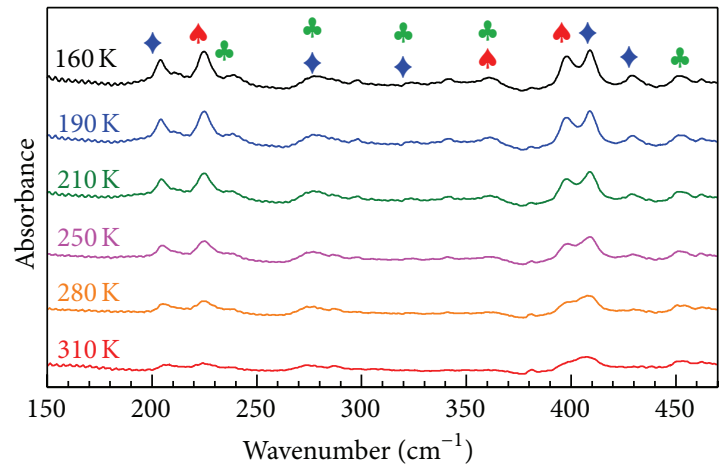

(e)

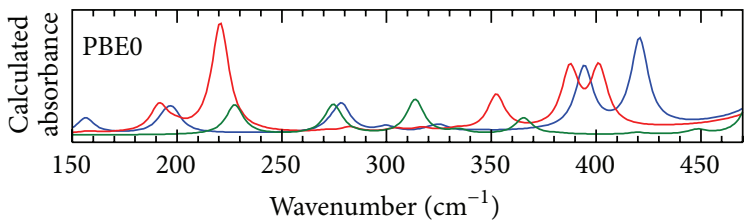

(d)

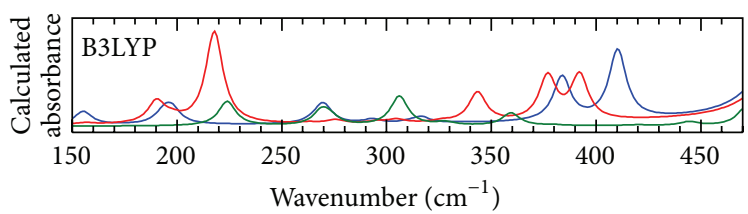

(c)

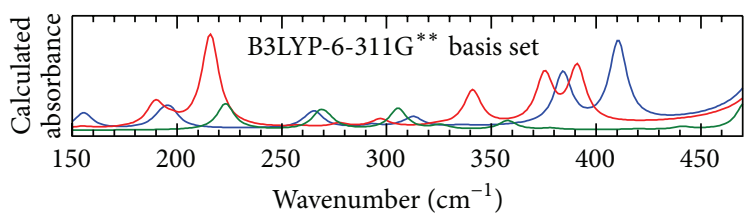

(b)

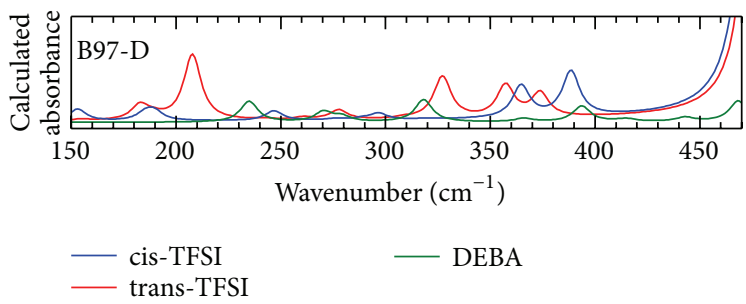

(a)

FIGURE 3: Measured infrared absorption of DEBA-TFSI as a function of temperature (panel (e), curves are stacked for clarity). The symbols refer to the attribution of the lines to trans-TFSI (red), cis-TFSI (blue), and cation (green) vibrations. For comparison purpose, the absorptions of TMPA-TFSI and TMHA-TFSI measured at $160 \mathrm{~K}$ are shown in panel (f) with the attribution of lines (same symbols used for DEBA-TFSI) [17]. In panels (a)-(d) absorptions of single ions in the frequency range between 150 and $460 \mathrm{~cm}^{-1}$ calculated with the various combinations of theories and basis sets are reported. No scaling factor was used. 


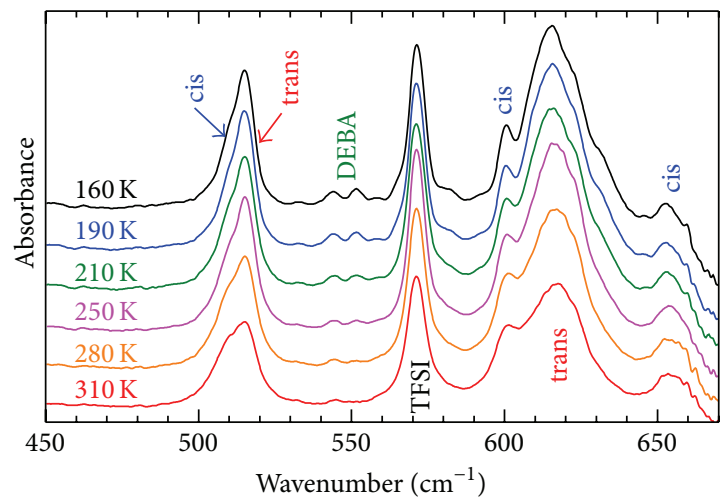

(e)

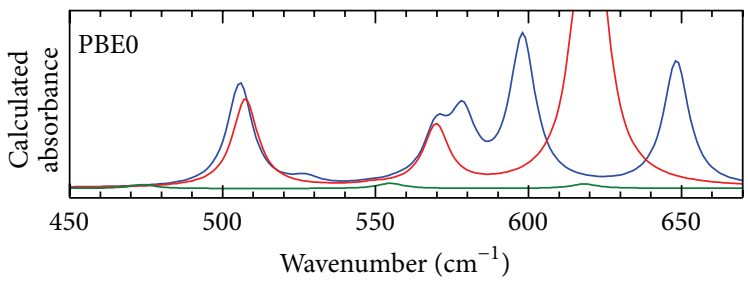

(d)

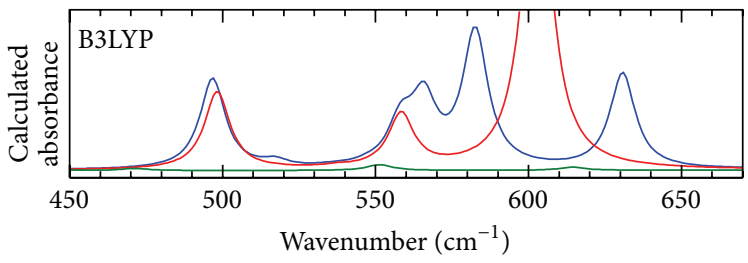

(c)

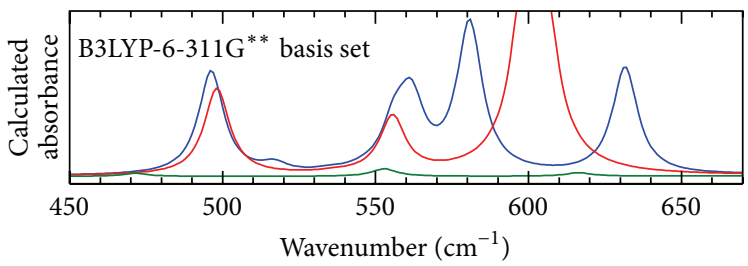

(b)

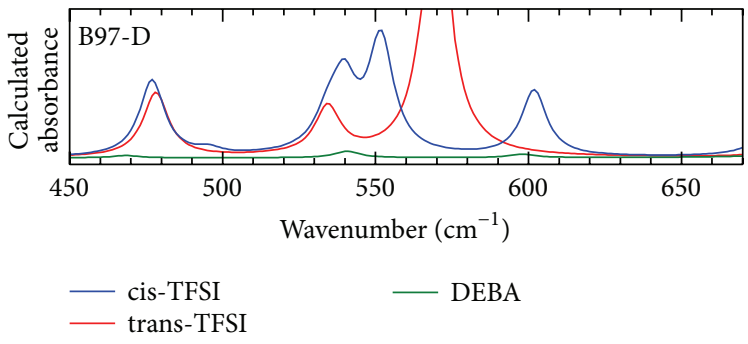

(a)

FIGURE 4: Measured infrared absorption of DEBA-TFSI as a function of temperature (panel (e), curves are stacked for clarity) and calculated absorption of single ions (panels (a)-(d)) in the frequency range between 480 and $665 \mathrm{~cm}^{-1}$. No scaling factor was applied.

basis sets in comparison with the experimental spectra in next section. However, it is well evident from Figures 3 and 4 that the calculated absorption spectra of each ion have similar spectral features, except that the vibration lines are generally displaced toward higher frequencies in the following order: B97-D/6-31G ${ }^{* *}, \mathrm{~B} 3 \mathrm{LYP} / 6-311 \mathrm{G}^{* *}, \mathrm{~B} 3 \mathrm{LYP} / 6-31 \mathrm{G}^{* *}$, and $\mathrm{PBE} 0 / 6-31 \mathrm{G}^{* *}$.
3.3. Infrared Spectroscopy Measurements: Attribution of the Vibration Lines. The experimental absorbance of DEBA-TFSI as a function of temperature is reported in Figures 3(e) and $4(\mathrm{e})$ in the spectral range between 170 and $470 \mathrm{~cm}^{-1}$ and 480 and $665 \mathrm{~cm}^{-1}$, respectively. We investigated this spectral interval as the markers of the presence of the two conformers of TFSI are centered there. In DEBA-TFSI, at 
TABLE 4: Experimental vibration frequencies at $160 \mathrm{~K}$ and attribution to ions.

\begin{tabular}{lc}
\hline Frequency $\left(\mathrm{cm}^{-1}\right)$ & Attribution \\
\hline 204.4 & cis-TFSI \\
224.4 & trans-TFSI \\
238.6 & DEBA \\
277.7 & DEBA/cis-TFSI \\
341.5 & DEBA/cis-TFSI \\
361.6 & DEBA/trans-TFSI \\
397.4 & trans-TFSI \\
409.6 & cis-TFSI \\
428.7 & cis-TFSI \\
451.2 & DEBA \\
509.97 & cis-TFSI \\
515.18 & trans-TFSI \\
544.2 & DEBA \\
551.4 & DEBA \\
571.2 & TFSI \\
582.0 & cis-TFSI \\
600.5 & cis-TFSI \\
615.6 & trans-TFSI \\
652.6 & cis-TFSI \\
\hline
\end{tabular}

$310 \mathrm{~K}$ one can observe broad lines centered around 206, 224, $275,398,408,451,509,515,571,600,618$, and $654 \mathrm{~cm}^{-1}$. At lower temperatures the spectrum becomes much more structured and at $160 \mathrm{~K}$ absorptions centered at 204.4, 224.4, 238.6, 277.7, 341.5, 361.6, 397.4, 409.6, 428.7, 451.2, 510.0, 515.2, $544.2,551.4,571.2,582.0,600.5,615.6$, and $652.6 \mathrm{~cm}^{-1}$ are clearly visible (Table 4 ). Some of those vibrations have already been attributed to the conformers of TFSI $[17,21]$. In fact a systematic investigation of the temperature dependence of the vibration lines of TMPA-TFSI and TMHA-TFSI allowed the attribution of the lines centered around 206, 288, 330, $406,435,603$, and $650 \mathrm{~cm}^{-1}$ to cis-TFSI and the attribution of the bands around $225,363,397$, and $622 \mathrm{~cm}^{-1}$ to trans-TFSI. Also lines around 510 and $571 \mathrm{~cm}^{-1}$ were ascribed to TFSI, even though in that case it could not be possible to separate the contributions of the two different conformers [17, 21]. Contributions coming from the vibrations of cations in some cases superimpose on the absorptions due to the anions, as reported in the upper part of Figure 3. In view of this previous study, the same attribution of the lines is here maintained.

The calculations performed on the DEBA ion suggest that it should possess vibration bands centered in the ranges 222/234, 267/278, 305/317, 357/365, 440/447, and $540 / 555 \mathrm{~cm}^{-1}$, depending on the particular choice of theory and basis set. Those bands have an intensity comparable to that of the TFSI bands, except the last one which is much weaker. Those absorptions should correspond to the lines found experimentally at 238.6, 277.7, 341.5, 361.6, and $451.2 \mathrm{~cm}^{-1}$ and to the couple of weak bands around 544 and $551 \mathrm{~cm}^{-1}$ bands. The lines between 277 and $379 \mathrm{~cm}^{-1}$ can contain a contribution from the TFSI ion. The frequency range between 480 and $665 \mathrm{~cm}^{-1}$ (Figure 4) is dominated by the absorptions of TFSI. The line at $571.2 \mathrm{~cm}^{-1}$ is common to both conformers of TFSI, while the absorption at $582.0 \mathrm{~cm}^{-1}$, visible in the low temperature spectra, can be ascribed to cis-TFSI. The intense vibrations with maxima at 600.5 and $652.6 \mathrm{~cm}^{-1}$ are certainly due to cis-TFSI, while the vibration at $615.6 \mathrm{~cm}^{-1}$ is attributable to trans-TFSI, as reported also by the previous literature [16-21]. In Figure 4, one can note that the line centered around $512 \mathrm{~cm}^{-1}$ is clearly composed of two absorptions centered at $\approx 510$ and $515 \mathrm{~cm}^{-1}$. In the previous literature, this vibration was attributed to TFSI, but it could not be possible to separate the contributions of the two conformers. DFT calculations already reported in the literature and the ones performed here agree in that the contribution of cis-TFSI should be centered at a slightly lower frequency than that of trans-TFSI [16-21]. In fact, independently of the combination of theory and basis sets used for calculations, the band due to cis-TFSI is centered at a frequency of $1-2 \mathrm{~cm}^{-1}$ lower than that of trans-TFSI (see Tables 1 and 2). In view of this, we here suggest that the lines centered around 510 and $515 \mathrm{~cm}^{-1}$ are due to cis-TFSI and trans-TFSI, respectively. The two contributions can be separated by a fit of the whole absorption line. In the previous literature similar fits were used to separate the contributions to the Raman spectra of the two conformers of TFSI centered around $740 \mathrm{~cm}^{-1}$, whose energies differ only by $3 \mathrm{~cm}^{-1}$ [18].

A remark concerns the comparison of the frequency position of the vibration lines calculated with different theories and basis sets: passing from $6-31 G^{* *}$ to $6-311 G^{* *}$ basis set, most lines are displaced only by $1-3 \mathrm{~cm}^{-1}$. Moving from B3LYP theory to B97-D functional, one obtains a drastic decrease of all frequencies by $7-10 \mathrm{~cm}^{-1}$ and in some cases even $20 \mathrm{~cm}^{-1}$. A mean scaling factor of $\sim 1.1$ should be used to accommodate calculated frequencies onto the experimental ones. Conversely, at the PBE0 level the frequencies increase by $2-15 \mathrm{~cm}^{-1}$ and a good agreement with the position of the experimental bands is found, even better than that obtained by the largely used B3LYP theory.

3.4. Temperature Dependence of the Concentration of the TFSI Conformers. Once the attribution of experimental absorptions to vibrations of ions is settled, we would like to study the temperature evolution of the concentration of the two conformers of TFSI. The ratio of the conformer concentration, $r$, in a liquid is proportional to the ratio of the intensities of the spectral lines attributable to specific conformers [16]. In our case we can distinguish two spectral ranges where we find close vibrations certainly attributable to the different conformers: (1) the lines centered around 510 and $515 \mathrm{~cm}^{-1}$ attributable to cis and trans TFSI, respectively, and (2) the line centered around $615 \mathrm{~cm}^{-1}$ due to transoid conformer and the absorptions around 600 and $650 \mathrm{~cm}^{-1}$ due to the cisoid conformer. Therefore, we define the ratio of the conformer concentration as

$$
r=\frac{\left[C_{\text {cis }}\right]}{\left[C_{\text {trans }}\right]}=\frac{I_{\text {cis }}}{I_{\text {trans }}},
$$

where $I_{x}$ designates the integrated IR intensity of the band centered at wavenumber $x$ [16], after subtraction of a linear 


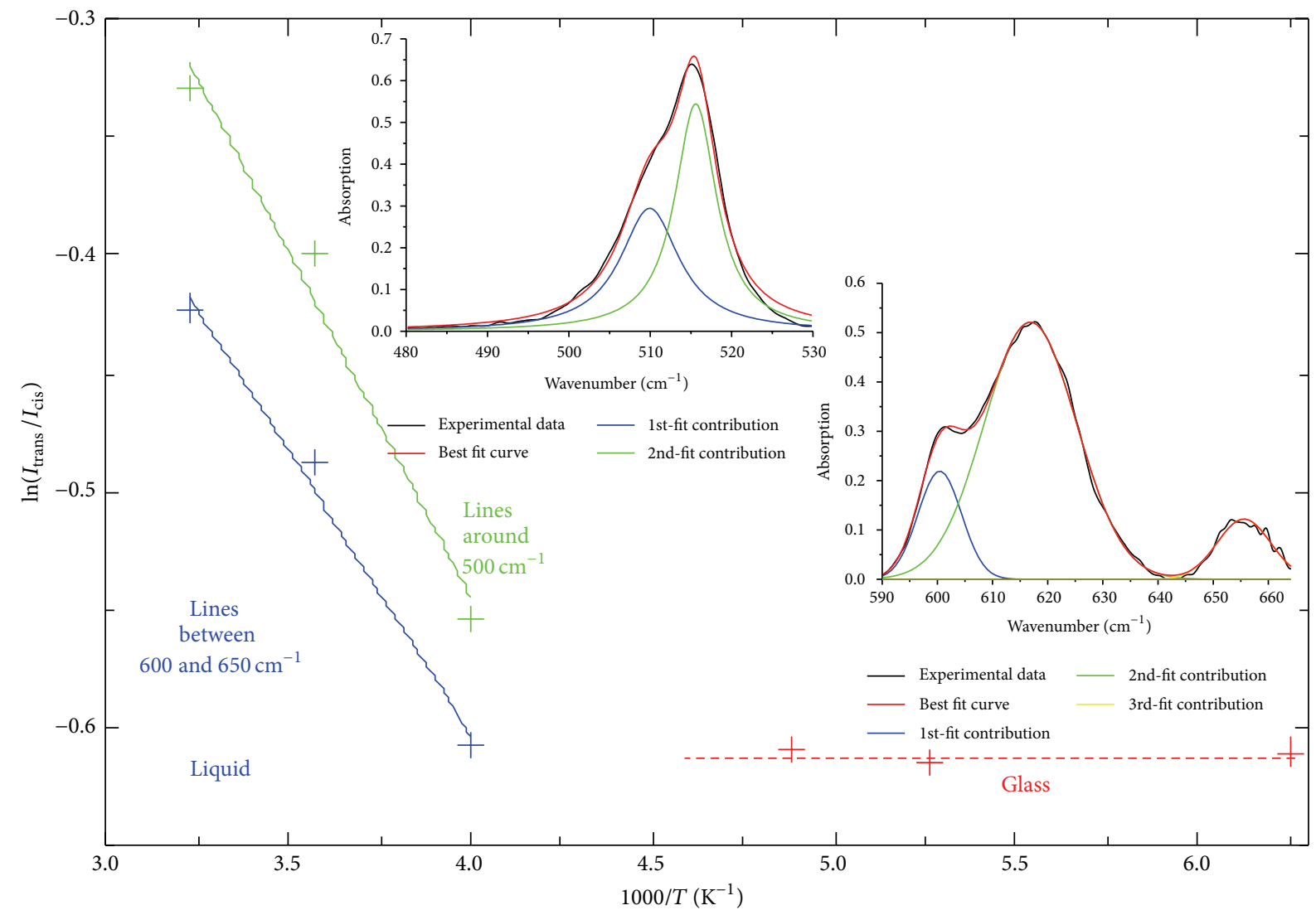

FIGURE 5: Temperature dependence of the ratio of the intensity of the vibration lines due to the transoid and cisoid conformers and best fit lines. In the inset two examples of the quality of the fit for the low frequency and high frequency lines are reported.

background from the absorption data reported in Figure 4. In the liquid state the following equation holds for the equilibrium constant, $K$, between conformers:

$$
-R T \ln K=\Delta H-T \Delta S \text {, }
$$

where $R$ is the gas constant, $T$ is the temperature, and $\Delta H$ and $\Delta S$ are the enthalpy and entropy difference of the conformers. Considering that the ratio between the conformer concentrations is proportional to the equilibrium constant $K \propto r$, one finally obtains that

$$
\ln (r)=-\frac{1}{T} \frac{\Delta H}{R}+\frac{\Delta S}{R}+c
$$

where $c$ is a constant [16]. In the case of the absorptions between 600 and $650 \mathrm{~cm}^{-1}$, we considered $I_{\text {cis }}=I_{600}+$ $I_{650}$ and $I_{\text {trans }}=I_{615}$. From a plot of $\ln (r)$ versus $1 / T$ one can verify the Arrhenius dependence of the concentration from temperature and it is possible to obtain the enthalpy difference between conformers. In Figure 5 one can note a straight linear dependence of $\ln (r)$ on $1 / T$ for our liquid. $\Delta H$ is found to be positive and equal to $2.4 \pm 0.4 \mathrm{~kJ} / \mathrm{mol}$ from these higher frequency lines. The same analysis has been performed considering the absorptions around $500 \mathrm{~cm}^{-1}$; that is, $I_{\text {cis }}=$ $I_{510}, I_{\text {trans }}=I_{515}$, and the value obtained for the enthalpy is $2.0 \pm 0.2 \mathrm{~kJ} / \mathrm{mol}$. Those results are well comparable within errors bars and, moreover, are in good agreement with the value of $2.2 \mathrm{~kJ} / \mathrm{mol}$ reported in the literature for the liquid state [16-21]. By the way, the coincidence of the two values corroborates our attribution to the different conformers of TFSI of the two lines centered around $500 \mathrm{~cm}^{-1}$.

In the liquid state, the ratio of the concentrations of the two TFSI conformers is governed by the Boltzmann factor, which depends on the enthalpy difference, $\Delta H$, between the two rotamers. In the liquid state, indeed there is a dynamic equilibrium, between TFSI ions in the two configurations. If no phase transition occurred, at low temperatures, the lowest energy conformer should become more populated; virtually for $T \rightarrow 0 \mathrm{~K}$, all TFSI ions should be in the transoid configuration. As temperature increases, the higher energy conformer should become more populated and, for $T \rightarrow \infty K$, the two energy states should be equally populated. Indeed, in our system this description is valid in the temperature range above $250 \mathrm{~K}$. However, on passing to the glassy state, the concentrations of the conformers deviated from the Boltzmann law and the relative concentration of two conformers remains practically constant below $\sim 210 \mathrm{~K}$ (see Figure 5). This is a clear spectroscopic indication of occurrence of the glass transition.

Indeed, infrared spectroscopy was used in the past to study the temperature evolution of the concentration of conformers in some polymers when entering the glassy state, 
such as poly(methylmethacrylate) (PMMA) [30] and poly(Llactide) (PLLA) [31]. In PMMA subtle temperature dependence of the population of trans and gauche conformers was reported and was used to determine the glass transition temperature [30]. Indeed, the fraction of gauche conformer abruptly increased above the glass transition temperature. Furthermore, it was found that molecular reorganization of PMMA occurs even below the glass transition temperature, $T_{g}$ [20]. In PLLA, near $T_{g}$, a change in the slope of the intensity of spectral lines versus temperature was detected [31]. It was suggested that the energy-favorable gauche-trans conformers are rearranged into the less energy-favorable gauche-gauche counterparts with heating over the glass transition region [31].

In the case of the presently investigated ionic liquid, we find that in the liquid phase the fraction of conformers as a function of temperature is governed by the Boltzmann distribution. On the contrary, in the glassy state a sudden freezing of the molecular configuration occurs.

\section{Conclusions}

A combined study of the ionic liquid N,N-Dimethyl-N-ethylN-benzylammonium bis(trifluoromethanesulfonyl)imide, by means of infrared spectroscopy and DFT calculations, allows the attribution of all the absorption lines occurring between 150 and $670 \mathrm{~cm}^{-1}$ to the vibrations of the single ions composing the liquid. The cation gives rise to five weak absorptions below $450 \mathrm{~cm}^{-1}$ and to two weak lines around $550 \mathrm{~cm}^{-1}$. On the other hand, the two conformers of TFSI possess strong vibration bands. For the first time, we are able to separate the two contributions of cis- and trans-TFSI at 510 and $515 \mathrm{~cm}^{-1}$. The temperature dependence of the ratio of the intensity of the vibrations of the lines assigned to cis-TFSI and transTFSI is proportional to the relative concentration of the two conformers. In the liquid state, the relative concentrations of the two conformers of TFSI depend on temperature through the Boltzmann factor and the energy separation, $\Delta H$, is found to $b e \approx 2 \mathrm{~kJ} / \mathrm{mol}$. On the contrary, in the glassy state a sudden freezing of the molecular configuration occurs. Moreover, we find that the vibration frequencies calculated by means of the PBE0 functional are in better agreement with the experimental ones than those calculated at the B3LYP level.

\section{Conflict of Interests}

The authors declare that there is no conflict of interests regarding the publication of this paper.

\section{Acknowledgments}

The authors wish to thank P. Roy and J.-B. Brubach for assistance at the AILES beamline of Synchrotron Soleil. This study was partially financed by the European Commission in the framework of the FP7 I3 CALIPSO Project for beamtime number 20131134 at Soleil Synchrotron (Contract no. 226716).

\section{References}

[1] N. V. Plechkova and K. R. Seddon, "Applications of ionic liquids in the chemical industry," Chemical Society Reviews, vol. 37, no. 1, pp. 123-150, 2008.

[2] T. Welton, "Ionic liquids in catalysis," Coordination Chemistry Reviews, vol. 248, no. 21-24, pp. 2459-2477, 2004.

[3] M. Armand, F. Endres, D. R. MacFarlane, H. Ohno, and B. Scrosati, "Ionic-liquid materials for the electrochemical challenges of the future," Nature Materials, vol. 8, no. 8, pp. 621-629, 2009.

[4] C. Chiappe and D. Pieraccini, "Ionic liquids: solvent properties and organic reactivity," Journal of Physical Organic Chemistry, vol. 18, no. 4, pp. 275-297, 2005.

[5] M. A. Navarra, "Ionic liquids as safe electrolyte components for Li-metal and Li-ion batteries," Material Research Society Bulletin, vol. 38, no. 7, pp. 548-553, 2013.

[6] A. Martinelli, A. Matic, P. Jacobsson, L. Börjesson, A. Fernicola, and B. Scrosati, "Phase behavior and ionic conductivity in lithium Bis(trifluoromethanesulfonyl)imide-doped ionic liquids of the pyrrolidinium cation and bis(trifluoromethanesulfonyl)imide anion," Journal of Physical Chemistry B, vol. 113, no. 32, pp. 11247-11251, 2009.

[7] F. M. Vitucci, D. Manzo, M. A. Navarra et al., "Low-temperature phase transitions of 1-butyl-1-methylpyrrolidinium bis(trifluoromethanesulfonyl)imide swelling a polyvinylidenefluoride electrospun membrane," Journal of Physical Chemistry C, vol. 118, no. 11, pp. 5749-5755, 2014.

[8] S. Jeremias, M. Carewska, L. Conte, S. Passerini, and G. B. Appetecchi, "Asymmetry effect of novel per(fluoroalkylsulfonyl)imide anions in pyrrolidinium ionic liquids," RSC Advances, vol. 3, no. 39, pp. 17755-17761, 2013.

[9] Y. Yoshimura, T. Takekiyo, Y. Imai, and H. Abe, "Pressureinduced spectral changes of room-temperature ionic liquid, $N, N$-diethyl- $N$-methyl- $N$-(2-methoxyethyl)ammonium Bis(trifluoromethylsulfonyl)imide, [DEME][TFSI]," Journal of Physical Chemistry C, vol. 116, no. 3, pp. 2097-2101, 2012.

[10] Y. Yoshimura, H. Abe, Y. Imai, T. Takekiyo, and N. Hamaya, "Decompression-induced crystal polymorphism in a roomtemperature ionic liquid, $\mathrm{N}, \mathrm{N}$-diethyl- $\mathrm{N}$-methyl- $\mathrm{N}$-(2methoxyethyl) ammonium tetrafluoroborate," Journal of Physical Chemistry B, vol. 117, no. 11, pp. 3264-3269, 2013.

[11] Y. Imai, T. Takekiyo, H. Abe, and Y. Yoshimura, "Pressureand temperature-induced Raman spectral changes of 1-butyl-3methylimidazolium tetrafluoroborate," High Pressure Research, vol. 31, no. 1, pp. 53-57, 2011.

[12] T. Takekiyo, N. Hatano, Y. Imai, H. Abe, and Y. Yoshimura, "Pressure-induced phase transition of 1-butyl-3-methylimidazolium hexafluorophosphate [bmim][PF6]," High Pressure Research, vol. 31, no. 1, pp. 35-38, 2011.

[13] L. F. O. Faria, M. M. Nobrega, M. L. A. Temperini, and M. C. C. Ribeiro, "Ionic liquids based on the bis(trifluoromethylsulfonyl)imide anion for high-pressure Raman spectroscopy measurements," Journal of Raman Spectroscopy, vol. 44, no. 3, pp. 481-484, 2013.

[14] O. Russina, B. Fazio, C. Schmidt, and A. Triolo, "Structural organization and phase behaviour of 1-butyl-3-methylimidazolium hexafluorophosphate: an high pressure Raman spectroscopy study," Physical Chemistry Chemical Physics, vol. 13, no. 25, pp. 12067-12074, 2011.

[15] L. Su, M. Li, X. Zhu et al., "In situ crystallization of low-melting ionic liquid $[\mathrm{BMIM}]\left[\mathrm{PF}_{6}\right]$ under high pressure up to $2 \mathrm{GPa}$," The 
Journal of Physical Chemistry B, vol. 114, no. 15, pp. 5061-5065, 2010.

[16] A. Martinelli, A. Matic, P. Johansson et al., "Conformational evolution of TFSI- in protic and aprotic ionic liquids," Journal of Raman Spectroscopy, vol. 42, no. 3, pp. 522-528, 2011.

[17] F. M. Vitucci, F. Trequattrini, O. Palumbo et al., "Stabilization of different conformers of bis(trifluoromethanesulfonyl)imide anion in ammonium-based ionic liquids at low temperatures," Journal of Physical Chemistry A, vol. 118, no. 38, pp. 8758-8764, 2014.

[18] M. Herstedt, M. Smirnov, P. Johansson et al., "Spectroscopic characterization of the conformational states of the bis(trifluoromethanesulfonyl)imide anion (TFSI-)," Journal of Raman Spectroscopy, vol. 36, no. 8, pp. 762-770, 2005.

[19] K. Fujii, T. Takamuku, R. Kanzaki, Y. Umebayashi, and S.I. Ishiguro, "Conformational equilibrium of bis(trifluoromethanesulfonyl) imide anion of a room-temperature ionic liquid: Raman spectroscopic study and DFT calculations," The Journal of Physical Chemistry B, vol. 110, no. 16, pp. 8179-8183, 2006.

[20] J. N. Canongia Lopes, K. Shimizu, A. A. H. Pádua et al., "A tale of two ions: the conformational landscapes of bis(trifluoromethanesulfonyl)amide and N,N-dialkylpyrrolidinium," Journal of Physical Chemistry B, vol. 112, no. 5, pp. 1465-1472, 2008.

[21] F. M. Vitucci, F. Trequattrini, O. Palumbo, J.-B. Brubach, P. Roy, and A. Paolone, "Infrared spectra of bis(trifluoromethanesulfonyl)imide based ionic liquids: experiments and DFT simulations," Vibrational Spectroscopy, vol. 74, pp. 81-87, 2014.

[22] K. Fujil, S. Seki, S. Fukuda et al., "Anion conformation of low-viscosity room-temperature ionic liquid 1-ethyl-3methylimidazolium bis(fluorosulfonyl) imide," Journal of Physical Chemistry B, vol. 111, no. 44, pp. 12829-12833, 2007.

[23] O. Palumbo, F. Vitucci, F. Trequattrini, and A. Paolone, "A study of the conformers of the $N, N$-diethyl- $N$-methyl- $N$ propylammonium ion by means of infrared spectroscopy and DFT calculations," Vibrational Spectroscopy, vol. 80, pp. 11-16, 2015.

[24] P. Roy, M. Guidi Cestelli, A. Nucara et al., "Spectral distribution of infrared synchrotron radiation by an insertion device and its edges: a comparison between experimental and simulated spectra," Physical Review Letters, vol. 84, no. 3, pp. 483-486, 2000.

[25] P. Roy, J.-B. Brubach, P. Calvani et al., "Infrared synchrotron radiation: from the production to the spectroscopic and microscopic applications," Nuclear Instruments and Methods in Physics Research Section A: Accelerators, Spectrometers, Detectors and Associated Equipment, vol. 467-468, part 1, pp. 426-436, 2001.

[26] Y. Shao, L. F. Molnar, Y. Jung et al., "Advances in methods and algorithms in a modern quantum chemistry program package," Physical Chemistry Chemical Physics, vol. 8, no. 27, pp. 3172-3191, 2006.

[27] W. J. Hehre, A Guide to Molecular Mechanics and Quantum Chemical Calculations, Wavefunction, Irvine, Calif, USA, 2003.

[28] A. A. Granovsky, Firefly version 8.0, http://classic.chem.msu.su/ gran/gamess/index.html.

[29] M. W. Schmidt, K. K. Baldridge, J. A. Boatz et al., "General atomic and molecular electronic structure system," Journal of Computational Chemistry, vol. 14, no. 11, pp. 1347-1363, 1993.

[30] H. S. Shin, Y. M. Jung, T. Y. Oh et al., "Glass transition temperature and conformational changes of poly(methyl methacrylate) thin films determined by a two-dimensional map representation of temperature-dependent reflection-absorption FTIR spectra," Langmuir, vol. 18, pp. 5953-5958, 2002.

[31] P. Pan, B. Zhu, T. Dong et al., "Conformational and microstructural characteristics of poly(L-lactide) during glass transition and physical aging," Journal of Chemical Physics, vol. 129, no. 18, Article ID 184902, 2008. 

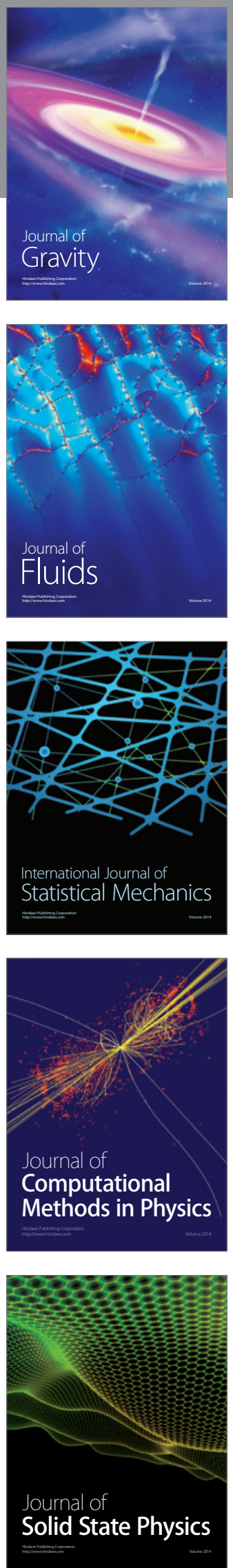

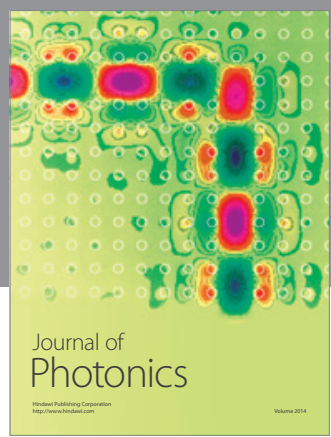

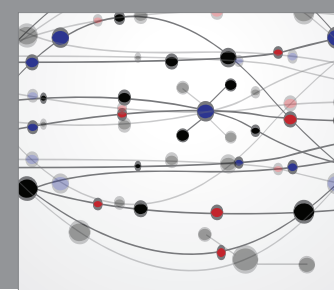

The Scientific World Journal

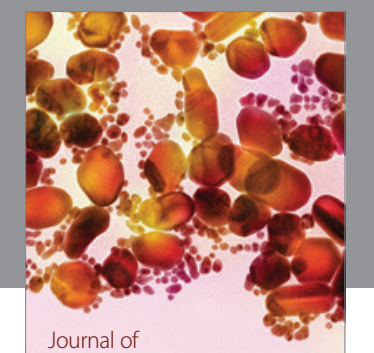

Soft Matter
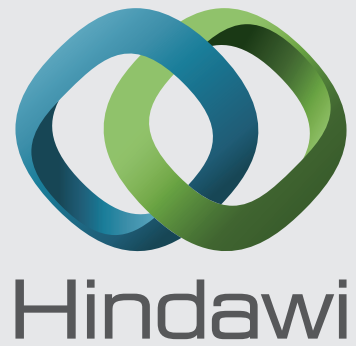

Submit your manuscripts at

http://www.hindawi.com
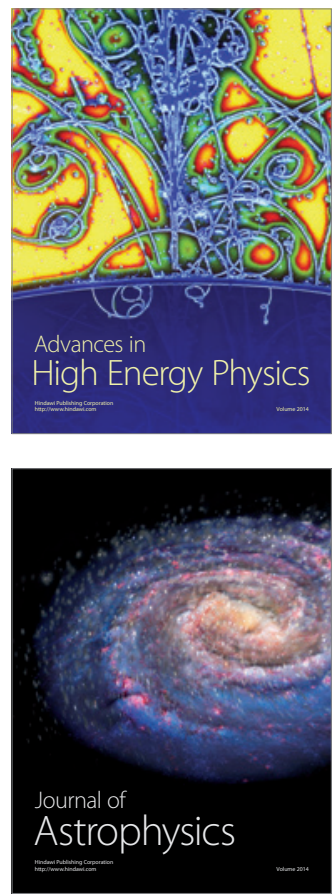
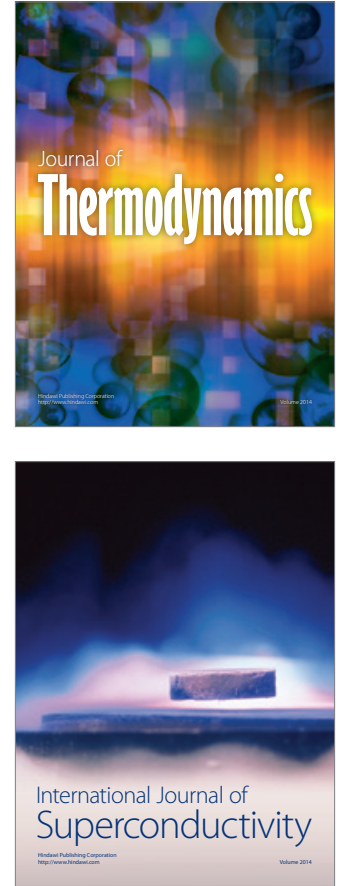
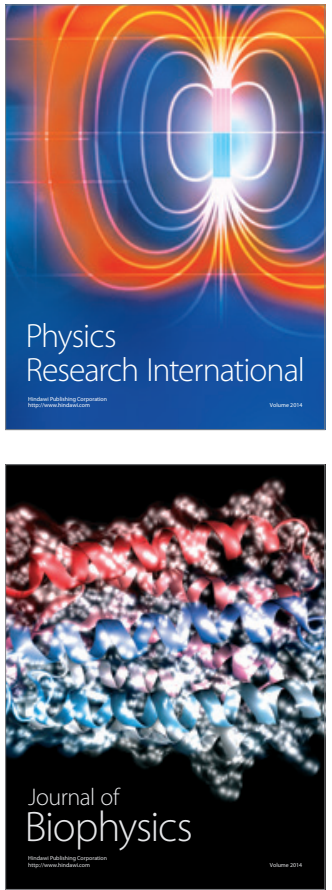
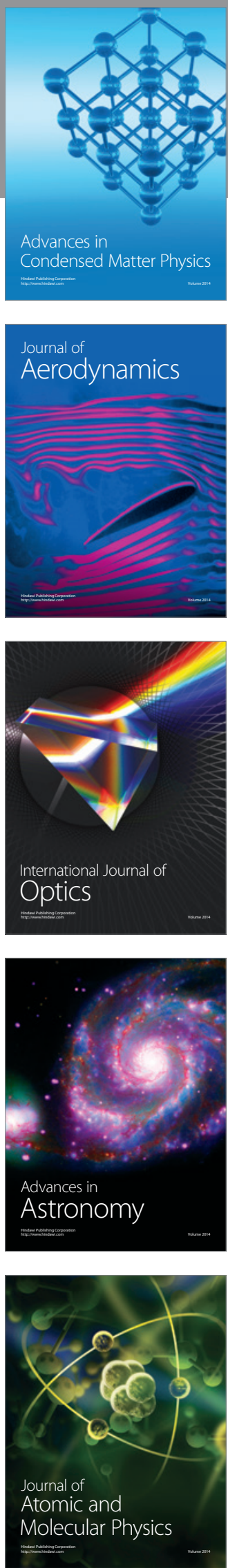